\title{
VASCULARIZACIÓN CORNEAL Y LENTES DE CONTACTO
}

\section{CORNEAL VASCULARISATION AND CONTACT LENSES}

\author{
PAPAS E $\mathrm{PhD}^{1}$
}

La córnea es avascular por la simple razón de que de otra forma comprometería su papel como la principal superficie refractiva del ojo. Como resultado, se intentan evitar de forma enérgica las situaciones que resultan en vascularización de este tejido bellamente transparente. La alteración visual provocada por la invasión vascular de la región corneal central es, quizá, la amenaza más obvia. Sin embargo, la vascularización corneal (VC) tiene otras consecuencias potencialmente lesivas que incluyen el poner en peligro la normal situación de privilegio inmune de la cámara anterior (1) y el aumentar el riesgo de rechazo del injerto $(2,3)$.

Para la población general, la situación más probable en la que se va a encontrar VC es en asociación con el uso de lentes de contacto. Aunque las estimaciones de prevalencia son bastante variables, se sugiere que entre un décimo y un tercio de todos los casos de VC implican el uso de lentes de contacto. Sin embargo, no todas las lentes son iguales a este respecto. Las cifras de prevalencia tienden a esconder el hecho de que las lentes rígidas gas permeables (RGP) tienen mucha menos probabilidad de estar asociadas a VC que las lentes de contacto blandas (LCB). Aunque no se conocen completamente los mecanismos por los que las lentes de contacto causan $\mathrm{VC}$, las respuestas divergentes a los varios tipos de lentes son muy instructivas y la consideración de las diferencias funcionales entre estas modalidades puede proporcionar una valiosa comprensión etiológica.

Los diámetros típicos de las lentes RGP están en la región de 9-10 $\mathrm{mm}$ y consiguientemente cubren sólo la porción central de la córnea durante su porte. Por el contrario, las LCB son notablemente mayores, alrededor de 13-15 mm, y cubren tanto la córnea entera como el limbo y parte de la conjunti- va perilímbica circundante. El efecto general de esta cobertura adicional con las LCB es el de reducir el acceso de los tejidos subyacentes al oxígeno de la atmósfera y al disuelto en la lágrima. Como resultado, la córnea periférica y el limbo probablemente experimentan un cierto grado de hipoxia. Una manifestación temprana de esto es la hiperemia de los vasos límbicos, una respuesta que ha sido reconocida durante muchos años durante el uso de LCB y que está directamente asociada con la hipoxia que estas lentes producen (4). Aunque hay debate en torno a si la hipoxia periférica es un estímulo suficiente en sí mismo para causar la VC, los cambios vasculares a corto plazo asociados son aparentemente idénticos a aquéllos vistos en córneas donde la VC termina por aparecer.

Otro aspecto fundamental del uso de lentes de contacto que incide en la VC es su modo de utilización. El uso de las lentes durante largos períodos de tiempo y particularmente durante los períodos de sueño, es decir, el uso prolongado o continuado, conlleva un mayor riesgo que el formato de uso diario convencional en el que las lentes se retiran antes de la oclusión del ojo. Las condiciones en el ojo cerrado son indicativas de un estado de inflamación subclínica, con incrementos importantes en el reclutamiento de leucocitos polimorfonucleares y la regulación a la alza de varios factores que tienen propiedades potencialmente angiogénicas (5). Bajo circunstancias normales, éstas están equilibradas por una regulación a la alza complementaria de factores angiostáticos, que mantienen así el statu quo. El uso de lentes de contacto parece tener la capacidad potencial de alterar este equilibrio y, de nuevo, la carga hipóxica aumentada puede ser el factor clave. Durante el sueño, la presión de oxígeno en la superficie anterior de las lentes de contacto se redu-

\footnotetext{
${ }^{1}$ Vision CRC, Instituto para la Investigación Oftalmológica, Escuela de Optometría y Ciencias de la Visión, Universidad de Nueva Gales del Sur, Sydney, Australia.

E-mail: E.Papas@ visioncrc.org
} 
ce desde los $155 \mathrm{mmHg}$ disponibles en la atmósfera hasta la proporcionada por los vasos de la conjuntiva palpebral, es decir, alrededor de $55 \mathrm{mmHg}$. El poner una lente de contacto entre el párpado cerrado y la córnea puede restringir aún más este aporte de oxígeno ya reducido.

Hasta hace poco, los materiales de las LCB no tenían capacidad suficiente para transmitir fisiológicamente niveles de oxígeno adecuados a la superficie ocular en todo el abanico de circunstancias que se presentan durante su uso. Por ello, las secuelas hipóxicas han sido frecuentes complicaciones del uso de LCB y estas secuelas incluyen la VC. Cuando el médico se enfrenta a la VC inducida con las LCB, su estrategia debe ser la de reducir la carga hipóxica. Tradicionalmente, esto significaba o bien reducir los períodos de porte o bien aumentar la oxigenación en la superficie ocular reduciendo el grosor de la lente y/o incrementado el contenido acuoso de la lente. Por supuesto siempre existe la opción de cambiar a lentes RGP, aunque esta opción es poco popular entre los portadores debido a la mayor incomodidad inicial y al período de adaptación más largo.

Recientemente, ha surgido una nueva alternativa importante gracias a la aparición de los materiales de hidrogel silicona. Estos nuevos polímeros permiten la fabricación de lentes de contacto con las mismas dimensiones y grado de comodidad de las LCB tradicionales, pero con mucha mayor capacidad de transmisión del oxígeno. El uso de estas lentes hace posible reducir la hipoxia durante su porte a niveles que, en muchos casos, se aproximan a los de una situación de no-uso de lente. Estos materiales pueden disminuir de forma significativa la aparición de VC durante el uso de LCB (6).

Aunque las lentes de contacto asociadas a VC podrían beneficiarse enormemente de la eliminación de la hipoxia, no sería razonable asumir que ésta es la única causa. Las lentes mal diseñadas o mal adaptadas pueden provocar lesión mecánica directa a la superficie ocular o, si se asocian a una pobre biocompatibilidad, provocar una disrupción grave de la película lagrimal. Si se mantiene de forma suficientemente prolongada, la lesión que esto crea puede ser suficiente para iniciar los aconteci- mientos de la cascada angiogénica. Es útil también recordar que mientras que la mayoría de los portadores de lentes de contacto lo hacen por motivos estéticos, hay un grupo importante que lo hace por necesidad terapéutica. Estos individuos requieren típicamente lentes especiales. Por ejemplo, condiciones como el queratocono, la miopía alta o la córnea irregular pueden precisar lentes esclerales y debido a su amplio diámetro y grosor estos tipos de lentes pueden suponer un reto fisiológico importante tanto a la superficie ocular como al encargado de adaptarlas. No es sorprendente, pues, que estos grupos experimenten VC más frecuentemente, o bien que la gravedad de esta $\mathrm{VC}$ sea relativamente mayor que la de la población con un uso estético (7). Una vez más, sin embargo, parecen conseguirse mejorías cuando se pueden utilizar materiales con alta transmisión de oxígeno, aunque el ámbito para hacerlo es actualmente relativamente limitado. Es de esperar que el desarrollo continuado de las propiedades de los materiales también podrá beneficiar a este grupo en un futuro próximo.

\section{REFERENCES}

1. Dana MR, Streilein JW. Loss and restoration of immune privilege in eyes with corneal neovascularization. Invest Ophthalmol Vis Sci 1996; 37: 2485-2494.

2. Price MO, Thompson RW Jr, Price FW Jr. Risk Factors for various causes of failure in initial corneal grafts. Arch Ophthalmol 2003; 121: 1087-1092.

3. Dandona L, Naduvilath TJ, Janarthanan M, Ragu K, Rao GN. Survival analysis and visual outcome in a large series of corneal transplants in India. Br J Ophthalmol 1997; 81: 726-731.

4. Papas EB. The role of hypoxia in the limbal vascular response to soft contact lens wear. Eye Contact Lens 2003; 29: $S 72-S 74$

5. Sack RA, Sathe S, Beaton A. Tear turnover and immune and inflammatory processes in the open-eye and closedeye environments: relationship to extended wear contact lens use. Eye Contact Lens 2003; 29: S80-S82.

6. Dumbleton KA, Chalmers RL, Richter DB, Fonn D. Vascular response to extended wear of hydrogel lenses with high and low oxygen permeability. Optom Vis Sci 2001; 78: 147-151.

7. Tan DT, Pullum KW, Buckley RJ. Medical applications of scleral contact lenses: 2. Gas-permeable scleral contact lenses. Cornea 1995; 14: 130-137. 